Proc. 3rd Int. Conf. Physics of Solid State Ionics (ICPSSI-3)

J. Phys. Soc. Jpn. 79 (2010) Suppl. A, pp. 25-28

(C) 2010 The Physical Society of Japan

\title{
Phonon Dispersion in Superionic Copper Selenide - Observation of Soft Phonon Modes in Superionic Phase Transition
}

\author{
Sergey A. DANILKIN, Mohana YETHIRAJ and Gordon J. KEARLEY
}

Bragg Institute, Australian Nuclear Science and Technology Organization, New Illawarra Road, Lucas Heights NSW, Australia

(Received October 23, 2009; accepted December 2, 2009)

This paper reports lattice dynamical measurements of $\mathrm{Cu}_{1.8} \mathrm{Se}$ superionic conductor in the superionic $\alpha$-phase at ambient temperature. Measurements of phonon dispersion curves were performed with the new triple-axis spectrometer, TAIPAN, at the OPAL reactor. We found that TA [100], TA [111] and $\mathrm{TA}_{1}$ [110] phonon branches demonstrate a decrease in frequency at wavevectors $q>0.5$ rather than the flattening observed previously. Results are compared with calculated density functional theoretical calculations showing the presence of unstable soft mode related to ordering of $\mathrm{Cu}$ atoms observed in $\mathrm{Cu}_{1.8} \mathrm{Se}$ at room temperature followed by $\alpha$ $\beta$ phase transition at a lower temperature. Superstructure arising from the ordering causes effects similar to the folding of the Brillouin zone, although phonon intensities at new Brillouin zone centres are weak. The coupling of low-energy phonon modes with displacement of mobile ions can explain the strong damping of phonons at $q>0.5$ observed in the experiment.

KEYWORDS: copper selenide, fast ion conductor, neutron scattering, lattice dynamics, phonon dispersion

\section{Introduction}

$\mathrm{Cu}_{2-\delta} \mathrm{Se}$ is a mixed ionic-electronic conductor with a superionic transition at $414 \mathrm{~K}$ at the stoichiometric composition. At room temperature the superionic $\alpha$-phase exists in the concentration range from $\delta=0.15$ to 0.25 . ${ }^{1}$. The characteristic features of copper selenide are the ordering of $\mathrm{Cu}$ atoms in the low-temperature phase and a random distribution of $\mathrm{Cu}$ over interstitial sites in high-temperature superionic phase. Lattice dynamics of $\mathrm{Cu}_{2-\delta} \mathrm{Se}$ are similar to other $\mathrm{Cu}$ and $\mathrm{Ag}$ fast ionic conductors showing the presence of low-energy excitations. The importance of low-lying modes that give the major contribution to thermal motion due to high density of states and low activation energy is widely recognised. In $\mathrm{Cu}$ chalcogenides the low-energy modes were originally observed in inelastic scattering experiments by Sakuma et al. $^{2)}$ in $\mathrm{Cu}_{2} \mathrm{Se}$ and assigned to localized vibrations. The ion plasma excitations ${ }^{3)}$ or transverse optic modes, as in anion conductors ${ }^{4)}$, may also be responsible for the low-energy peak in density of states. To clarify the situation measurements of the phonon density of states have been performed in $\mathrm{Cu}_{1.78} \mathrm{Se}$ and $\mathrm{Cu}_{2} \mathrm{Se}$ powder samples using a multidetector time-of flight spectrometer. ${ }^{5)}$. We observed that the intensity of the low-energy peaks changes strongly with scattering angle. Such a dependence on wave vector, $Q$, is characteristic for the dynamic structure factor of acoustic phonons. An acoustic origin of the low-energy mode was confirmed by inelastic neutron scattering measurements with a $\mathrm{Cu}_{1.85}$ Se single crystal sample. ${ }^{5}$. It was found that the measured dispersion curves of the transverse acoustic (TA) phonons show a drastic change in the slope and flatten out at phonon wave vectors $q>0.4$ with an energy of about $4 \mathrm{meV}$. At the same time phonon peaks show a strong broadening at these wave vectors. Several mechanisms can cause the phonon width to increase such as the coupling of acoustic phonons with the motions of mobile ions or hybridization of acoustic and optical phonons.

In this paper we present results of measurements of phonon dispersion curves in a $\mathrm{Cu}_{1.8}$ Se single crystal. Section 2.1 provides the experimental details and experimental data. Section 2.2 deals with model calculation of phonon dispersion modes and a discussion, followed by the conclusion in $\S 3$.

\section{Results and Discussions}

\subsection{Experimental}

Measurements of phonon dispersion curves were performed on a single-crystal sample of $\mathrm{Cu}_{1.8}$ Se which has the structure of the superionic $\alpha$-phase at room temperature. The sample had a cylindrical shape with $12 \mathrm{~mm}$ diameter and $40 \mathrm{~mm}$ height. It was prepared by solid-state reaction of high purity $\mathrm{Cu}$ and Se powders in evacuated sealed quartz ampoules. The mixed components were heated to $720 \mathrm{~K}$ for about $100 \mathrm{hrs}$ with subsequent annealing for $48 \mathrm{hrs}$ at $420-520 \mathrm{~K}$. This crystal has an antifluorite structure with lattice parameter of $5.76 \AA$, with a mosaic spread of about 30 minutes and cylinder axes close to the [100] direction.

The neutron inelastic scattering experiment was carried out with the TAIPAN thermal triple axis spectrometer ${ }^{6}$ with a vertically focused HOPG (002) monochromator and a flat HOPG analyzer. A fixed final neutron energy of $14.87 \mathrm{meV}$ and $40^{\prime}$ collimations before and after the sample were used in the experiment. A PG filter was placed after the sample to suppress contribution from high order reflections. The sample was aligned in HK0 and HHL scattering planes. We measured phonon dispersion curves in [100], [110] and [111] directions. Due to strong broadening of phonon peaks data are limited to low energy modes. The onset of the broadening is rapid and takes place at phonon wavevectors $q$ $\approx 0.5$ for all measured phonon branches. This is clearly seen in Figs. 1(a) and 1(b) where typical $q$-dependence of phonon peaks is shown for $\mathrm{TA}_{1}$ [110] branch. Phonons with wave 

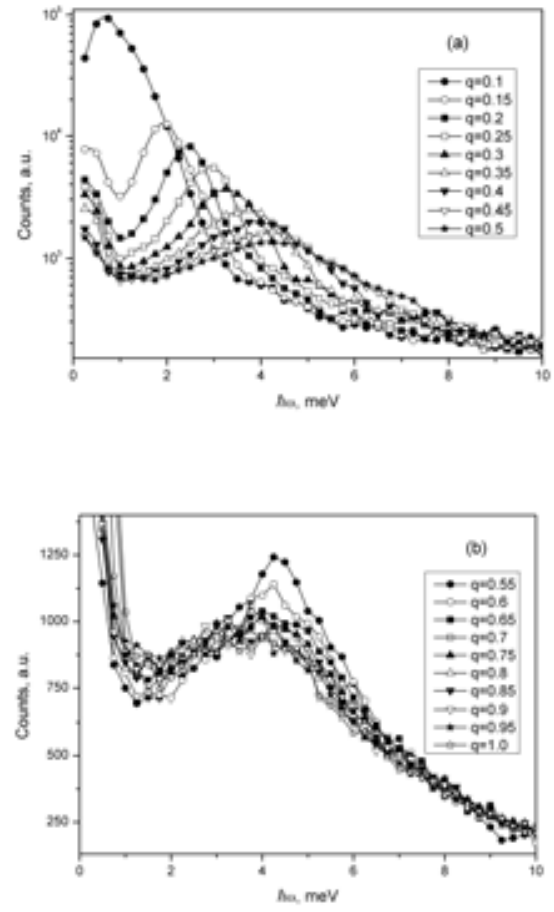

Fig. 1. (Color online) One-phonon peaks in $\mathrm{Cu}_{1.8} \mathrm{Se}$ corresponding to the transverse acoustic $\mathrm{TA}_{2}$ branch with wave vector, $q$, along [110] and polarization vector [1-10].

(a) Phonons wavevectors $q \leq 0.5$, logarithmic y-scale

(b) Phonons wavevectors $q \geq 0.5$, linear y-scale.

(rlu - reciprocal lattice unit; $1 \mathrm{rlu}=2 \pi / a, a$ - parameter of crystallographic unit cell)

vectors $q \leq 0.5$ (Fig. 1(a), logarithmic scale) are narrow and show a rapid decrease in intensity with increasing wave vector which is characteristic for acoustic phonons. At higher wave vectors $q \geq 0.5$ (Fig. 1b, linear scale) a change-over from strong $q$-dependence to minor modifications in peak shape and intensity takes place. Such behaviour makes the analysis of broad phonon peaks difficult because the phonon frequency alone does not represent the data adequately. This should be taken into account for dispersion curves shown in Fig. 2 where the frequencies of phonon peaks at $q \geq 0.5$ relate to centre of broad peaks without corrections. In general the present data (Fig. 2) agree well with our previous results. ${ }^{5)}$. Since the new data cover a wider $q$-range and measured with higher accuracy we found that the $\mathrm{TA}_{1}$ [110] branch with the polarization vector along [1-10] direction and TA phonons in [100] direction demonstrate a decrease in frequency at $q \geq$ 0.5 rather than the flattening observed previously. The transverse acoustic branch with wave vector in the [111] direction shows a considerably greater decrease for $q \geq 0.25$. In contrast, the $q$-dependence of frequency of $\mathrm{TA}_{2}$ [110] phonons polarized in [001] direction shows almost linear behaviour.

\subsection{Model calculations}

Phonon dispersion curves were calculated with density functional theoretical (DFT) approach. ${ }^{7-9)}$ In order to determine the force constants the structure has to be at the energy minimum, which corresponds to the zero-Kelvin structure. This was achieved by energy calculations and structural optimization using the VASP5.2 package, with the Perdew - Burke - Ernzerhof (PBE) exchange - correlation functional and projector augmented wave (PAW) pseudo-potentials with an energy cutoff of $600 \mathrm{eV}$. The energy-minimised structure is only slightly different from the measured structure. Calculations were performed for stoichiometric composition and antifluorite crystal structure with lattice parameter of $5.76 \AA$. A $2 \times 2 \times 2$ supercell of the crystallographic unit cell was used for all calculations, with the reciprocal lattice being sampled using $8 \quad q$-points. Single-point energy calculations were made for a series of structures in which the crystallographically distinct atoms were displaced by $0.03 \AA$ in positive and negative directions along the $x, y$ and $z$ directions. These calculations gave the Hellmann - Feynmann forces acting on atom and were used as input for the lattice-dynamics program, PHONON 4.2.4. ${ }^{10) .}$ The software package PHONON 4.2.4 was used to calculate the eigenfrequencies, eigenvectors and dispersion curves.

Calculated dispersion curves are shown in Figure 2 together with experimental data. The initial slope which is a measure of the sound velocity is in very good agreement with the DFT calculations. However, in [111] direction at approximately $0.1 \mathrm{rlu}$ the phonon branch has a softening somewhat reminiscent of a Kohn anomaly. This occurs for both the transverse and longitudinal acoustic branches, but only for phonons propagating along the [111] direction. Sound velocities in the other directions are in excellent agreement with the calculations. Calculated longitudinal modes cross the low-energy optic modes at wavevectors close to midpoint between the edge and the centre of Brillouin zone (BZ). Above this $q$-value and phonon energy of $10-14 \mathrm{meV}$, sharp longitudinal acoustic (LA) phonon



Fig. 2. Phonon dispersion curves in $\mathrm{Cu}_{1.8} \mathrm{Se}$.

Dots - experiment, lines - calculations. Arrows show the position of superstructural reflections.

peaks are no longer visible when measured in constant- $E$ mode.This sharp change in slope of dispersion curves probably can reflect hybridization with optical modes. The flattening of longitudinal modes at $q \geq 0.5$ is clearly seen in [100] and [110] direction diving rise to pronounced band at 12 - $14 \mathrm{meV}$ in phonon density of states. The most remarkable features of calculated acoustic modes are the low frequencies and the instability over a large area of reciprocal 
space. Acoustic modes TA [100], TA [111], TA 1 [110] and LA [110] are unstable and go to negative values in vicinity of BZ centre and at $q \geq 0.3-0.4$ in case of transverse modes and at $K$ - point for LA [110] mode. This indicates that the stoichiometric compound is dynamically unstable and antifluorite structure is not true low-temperature one. Indeed, a phase transition to low-temperature $\beta$-phase takes place at $414 \mathrm{~K}$ for stoichiometric $\mathrm{Cu}_{2} \mathrm{Se}$. ${ }^{1)}$.

The instability of acoustic modes is directly related to the order - disorder transformations observed in copper-selenide. Previously, in neutron diffraction experiments, we observed the superstructure reflections in $\mathrm{Cu}_{1.8} \mathrm{Se}$ single crystal at ambient temperature. ${ }^{11)}$. Reflections were found at the $\mathrm{G} \pm$ $1 / 2<111>$ and $G \pm 1 / 3<220>$ positions of reciprocal space in agreement with data of Kashida and Akai ${ }^{12)}$ for the low-temperature $\beta-\mathrm{Cu}_{2} \mathrm{Se}$ phase at room-temperature. Note that we observed ordering in the $\mathrm{Cu}_{1.8}$ Se compound which is described as "disordered" $\alpha$ - phase in the literature. ${ }^{1)}$. On heating we found that superstructural peaks disappear gradually and vanish at $\sim 365 \mathrm{~K}$. The intensity of the superstructure reflections is quite large. It can be seen that in the case of the $(2.52 .51 .5)$ reflection (Fig. 3) the intensity is comparable with that of the (222) Bragg peak. The appearance of strong reflections at the edge of the BZ in this direction can cause effects similar to the folding of the Brillouin zone. Indeed, both Figs. 2 and 3 show that TA phonon branch in [111] direction has tendency to soften at the boundary of BZ, although phonon intensities at "new" (2.5 2.5 1.5) BZ centre are weak. In Fig. 2 the positions of superstructural reflections are shown by arrows at $L$-point and at $q=0.66$ in direction [110] which is close to $K$-point. These points are in the area of pronounced instability of acoustic modes indicating that ordering process is actually driven by a soft mode. The effect of folding of the BZ has direct relationship to the suggestion of Wakamura who considers that the low-energy mode in $\beta$-AgI originates from the zone-edge acoustic phonons in $\gamma$-AgI because of folding of BZ. ${ }^{13)}$. However, the important difference in the case of the $\mathrm{Cu}_{1.8} \mathrm{Se}$ compound is that the ordering process and the folding of the BZ are driven by a soft mode, that is a second order transition compared to a first order transition as found in AgI.

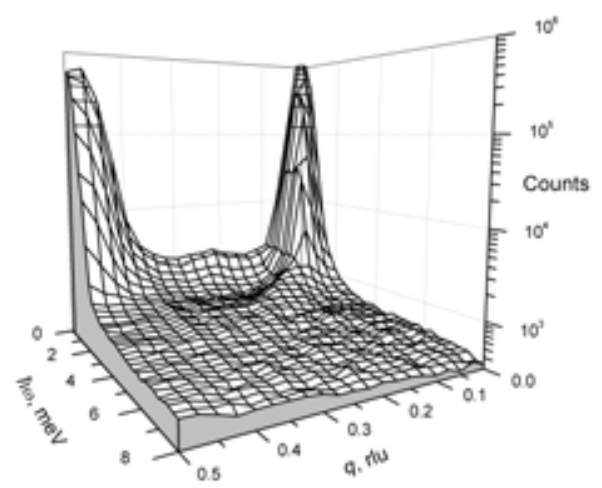

Fig. 3. (Color online) Surface plot of inelastic scattering intensity in $\mathrm{Cu}_{1.8}$ Se determined from a series of constant $-Q$ scans along direction $[2+q, 2+q, 2-q]$. Corresponds to the TA [111] phonon-dispersion branch.
As already noted, optical modes were not detected in current experiment and therefore we will compare our calculations with density of states measured on powder samples. According to paper ${ }^{14)}$ the frequency spectra of $\mathrm{Cu}_{2} \mathrm{Se}$ and $\mathrm{Cu}_{1.75} \mathrm{Se}$ have maxima at 13 and $25 \mathrm{meV}$ and boundary at $\sim 27 \mathrm{meV}$. As noted the lower maximum originates from almost dispersionless optic branches with energies in a range $12-13 \mathrm{meV}$ and flat parts of acoustic modes (Fig. 2). Optic modes with higher energies are also nearly flat at $q \geq 0.5$ giving rise to the second maximum in phonon density of states. The high energy boundary of the experimental spectrum is in agreement with the calculated value of $26 \mathrm{meV}$.

Acoustic phonon peaks at $q \geq 0.5$ are very broad in all directions that were studied. The width of the peaks is comparable with the energy of phonons, which can be an effect of coupling of phonons with displacements of mobile ions. Notice that "softened" transverse branches have a polarization vector in the direction $\langle 110\rangle$, orthogonal to the $<111>$; the $<111>$ directions which is assumed to be diffusion path for $\mathrm{Cu}$ atoms. ${ }^{15}$ ). Such vibrational modes can promote the diffusion of mobile ions.

\section{Conclusion}

We found that transverse acoustic phonons with wavevectors in [100], [111] and $\mathrm{TA}_{1}$ [110] branch have tendency to soften at BZ boundary. DFT calculations show that these modes are unstable over the large area in $Q$ space. This directly correlates with the observation of ordering in $\mathrm{Cu}$ sub-lattice in the $\alpha$-phase at ambient temperature. The superstructure arising from the ordering causes effects similar to the folding of the BZ, when new phonon branches arise from new BZ centres. The coupling of low-energy phonon modes with displacement of mobile ions is particularly relevant to the superionic conductivity and this may explain the strong damping of phonons at $q>0.5$ observed in experiment.

\section{Acknowledgment}

These experimental data are amongst the first to be recorded with the new instrument TAIPAN and we would like to take this opportunity to that all those who have been involved with the construction of this instrument. The authors would like to thank N.N. Bickulova from Sterlitamak University for synthesizing of crystal studied in this work.

1) N. H. Abrikosov, V. F. Bankina, M. A. Korzhuev, G. K. Demenski and O .A. Teplov: Sov. Phys. - Solid State 25 (1983) 1678.

2) T. Sakuma: Bulletin of Electrochemistry 11 (1995) 57.

3) M. Kobayashi, T. Tomoyose and M. Aniya: J. Phys. Soc. Jpn. 60 (1991) 3742.

4) W. Bührer and P. Brüesch: Solid State Communs. 16 (1975) 155.

5) S. A. Danilkin, A. N. Skomorokhov, A. Hoser, H. Fuess, V. Rajevac and N. N. Bickulova: J. Alloys and Compounds 361 (2003) 57.

6) S. Danilkin, G. Horton, R. Moore, G. Braoudakis and M. Hagen: J. of Neutron Research 15 (2007) 55.

7) G. Kresse and J. Furthmüller: Software VASP, Vienna (1999).

8) G. Kresse and J. Furthmüller: Phys. Rev. B 54 (1996) 11169. 
9) G. Kresse and J. Furthmüller: Comput. Mat. Sci. 6 (1996) 15

10) K. Parlinski, Z. Q. Li and Y. Kawazoe: Phys. Rev. Lett. 78 (1997) 4063.

11) S. A. Danilkin: Solid State Ionics 180 (2009) 483.

12) S. Kashida and J. Akai: J. Phys. C: Solid State Phys. 21 (1988) 5329.
13) K. Wakamura: Phys. Rev. B 59 (1999) 3560.

14) N. N. Bickulova, S. A. Danilkin, V. A. Semenov, E. L. Yadrovski, Z. A. Yagapharova and M. Y. Gareeva: Bulletin of the Bashkir State University 1 (2000) 33.

15) K. Yamamoto and S. Kashida: Solid State Ionics 48 (1991) 241. 